\title{
Expression and phosphorylation of stathmin correlate with cell migration in esophageal squamous cell carcinoma
}

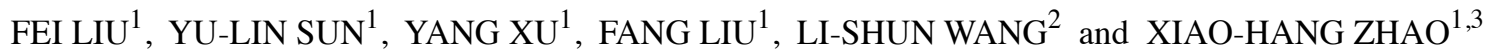 \\ ${ }^{1}$ State Key Laboratory of Molecular Oncology, Cancer Institute and Hospital, Chinese Academy of Medical Sciences \\ and Peking Union Medical College, Beijing 100021; ${ }^{2}$ Beijing Genomics Institute, Chinese Academy of Sciences, \\ Beijing 101300; ${ }^{3}$ Center of Basic Medical Sciences, Navy General Hospital, Beijing 100048, P.R. China
}

Received February 17, 2012; Accepted May 21, 2012

DOI: $10.3892 /$ or.2012.2157

\begin{abstract}
Microtubules play extensive roles in cellular processes, including cell motility. Stathmin is an important protein which destabilizes microtubules. The essential function of stathmin is closely associated with its phosphorylation status. Stathmin is overexpressed in many human cancers and has a significant relationship with clinical characteristics such as grade, tumor size and prognosis. We demonstrated that stathmin was overexpressed in ESCC tissues using both 2-DE and immunohistochemistry analysis. In addition, overexpression of stathmin was significantly correlated with histological grade in ESCC. However, no correlation was found with age, gender and lymph node metastasis. Knockdown of stathmin with siRNA impaired cell migration in KYSE30 and KYSE410 cells. When EC0156 cells were treated with paclitaxel, stathmin was stably phosphorylated and migration was impaired. These observations suggest that stathmin may have a more important function in ESCC development and migration. The present study provides further understanding of the importance of stathmin in ESCC therapy or diagnosis.
\end{abstract}

\section{Introduction}

Esophageal cancer (EC) is the eighth most common cancer worldwide (1), while esophageal squamous cell carcinoma (ESCC) is the predominant histological subtype in Asia, especially in China. It is characterized by high incidence and mortality rate $(1,2)$. We found that stathmin is a differentially expressed protein between cancer and adjacent normal tissues in ESCC using proteomic technology.

Correspondence to: Professor Xiao-Hang Zhao, Center of Basic Medical Sciences, Navy General Hospital and State Key Laboratory of Molecular Oncology, Cancer Institute and Hospital, CAMS \& PUMC, Beijing 100021, P.R. China

E-mail: zhaoxh@cicams.ac.cn

Key words: stathmin, esophageal squamous cell carcinoma, migration, phosphorylation
Stathmin is an important protein which destabilizes microtubules $(3,4)$. Microtubules are essential for many cellular processes, including mitosis, intracellular transport, supportment of cell shape and cell motility, and stathmin playes an important role in the regulation of microtubule which was involved in the construction and function of the mitotic spindle (5). Phosphorylation of stathmin led to a loss of the microtubule-destabilizing activity (6-8). Inhibition of stathmin phosphorylation produced strong mitotic phenotypes characterized by disassembly and disorganization of mitotic spindles and abnormal chromosome distributions (6). Stathmin phosphorylation gradient was necessary for correct spindle formation. Gradients of diffusible morphogens are known to be crucial for the supracellular self-organization of tissues and organisms (9).

Many studies had reported that stathmin was overexpressed across a broad range of human cancers, including acute leukemia, lymphoma, neuroblastoma and ovarian, prostate, breast and lung cancer (10-12).

The upregulation of stathmin in ESCC was reported and associated with differentiation degree, lymph node metastasis, invasive depth and TNM stage $(13,14)$. Wang et al demonstrated that knockdown of stathmin by antisense oligonucleotide can inhibit the proliferation of ECa109 cells (15). The expression and exact biological function of stathmin in ESCC, especially motility, remained largely unclear.

\section{Materials and methods}

Cell culture. ESCC cell lines EC0156 was established by our laboratory (16). KYSE30, KYSE140, KYSE150, KYSE170, KYSE180, KYSE410 and KYSE510 were donated by Dr Y. Shimada. EC0156 was cultured in Dulbecco's modified Eagle's medium (HyClone, UT, USA) supplemented with $10 \%$ fetal calf serum, $100 \mathrm{U} / \mathrm{ml}$ penicillin and $100 \mu \mathrm{g} / \mathrm{ml}$ streptomycin (Gibco, NY, USA). The other cell lines were cultured in RPMI-1640 medium supplemented with $10 \%$ heat-inactivated fetal bovine serum. All the cells were incubated at $37^{\circ} \mathrm{C}$ in a humidified atmosphere of $5 \% \mathrm{CO}_{2}$.

Tissue specimens. From January 1999 to 2002, 8 pairs of specimens for two dimensional electrophoresis were obtained 
from surgically resected ESCC tissues in Cancer Hospital of Chinese Academy of Medical Sciences (CAMS). Another 50 tissues specimes for immnohistochemistry were also obtained from surgically resected esophageal carcinoma in Cancer Hospital of CAMS from January 1999 to 2009. Tissue specimens $(\mathrm{n}=93)$ for immunohistochemistry were purchased as microarray (Outdo Biotech Co., Shanghai, China). All specimens were frozen immediately, stored at liquid nitrogen. The median age of the patients of the 143 ESCC tissues for immunochemistry was 60 years (range, 29-84 years).

Protein extraction and quantification. Approximately $1 \times 10^{7}$ cells were grown to $80 \%$ confluence and washed six times in $1 \mathrm{X}$ phosphate-buffered saline (PBS). Soluble proteins were extracted with lysis $(50 \mathrm{mM}$ Tris- $\mathrm{HCl} \mathrm{pH} 7.4,150 \mathrm{mM}$ $\mathrm{NaCl}, 1 \%$ Triton X-100, $0.1 \%$ SDS) with protease inhibitor cocktail (2.5 $\mu \mathrm{M}$ AEBSF, $0.04 \mu \mathrm{g} / \mathrm{ml}$ aprotinin, $0.04 \mu \mathrm{g} / \mathrm{ml}$ leupeptin, $1 \mathrm{mM}$ EDTA ) by super-sonication and followed by centrifugation at $12,000 \mathrm{~g}$ for $15 \mathrm{~min}$. Protein concentration was measured by the Bradford method.

Two dimensional electrophoresis. The soluble proteins from individual tissue specimen and the pooled tissue samples were separated by two dimensional electrophoresis (2-DE). Commercial IPG strips (pH 3-10NL, $18 \mathrm{~cm}$; Amersham Biosciences, Uppsala, Sweden), were rehydrated overnight with $450 \mu 1$ solution containing $8 \mathrm{M}$ urea, $2 \%$ w/v CHAPS, $20 \mathrm{mM}$ DTT, $0.5 \% \mathrm{v} / \mathrm{v}$ IPG buffer, $0.002 \%$ bromophenol blue and $1000 \mu \mathrm{g}$ protein. Electrofocusing was carried out for $60 \mathrm{kVh}$ at $20^{\circ} \mathrm{C}$ following the manufacturer's instruction. Prior to the second dimension, the IPG strips were equilibrated for 30 min with $50 \mathrm{mM}$ Tris- $\mathrm{HCl} \mathrm{pH} 8.8,6 \mathrm{M}$ urea, 30\% $\mathrm{v} / \mathrm{v}$ glycerol, $2 \% \mathrm{w} / \mathrm{v}$ SDS, and a trace of bromophenol blue followed by reduction with $1 \%$ of DTT and alkylation with $2.5 \%$ of iodoacetamide. The IPG strips were placed into $12 \%$ SDS-polyacrylamide gels $(26 \times 20 \mathrm{~cm})$ and were further electrophoresed by an EttanII-DE system (Amersham Biosciences) with a programmable power control, $0.5 \mathrm{~h}$ at $0.5 \mathrm{~W}$ per gel, then at $15 \mathrm{~W}$ per gel until the dye front reached the gel bottom. The separated proteins were visualized by Coomassie Brilliant Blue staining.

Protein identification. Briefly, images of the stained gels were acquired with an Image Scanner (Amersham Biosciences) using transmissive light. The gel images were first analyzed by eyes and subsequently were analyzed by ImageMaster 2D Elite 4.01 (Amersham Biosciences). Protein spots with signals differential intensity reaching 2.0 in $2 \mathrm{D}$ gels were excised, then were digested in modified trypsin solution with a final substrate-to-trypsin ratio of 40:1 (W:W) (in $25 \mathrm{mM}$ ammonium bicarbonate). The digested peptides from 2-DE gel spots were analyzed by MALDI-TOF-MS using an Ettan-MALDI-TOF system (Amersham Biosciences). Monoisotopic peptide masses obtained from MALDI-TOF were used to search the NCBInr protein database using Mascot algorithm.

Immunohistochemistry. For immunohistochemical staining multiple tissue arrays (MTA) of formalin-fixed and ESCC and their matched adjacent normal tissues were incubated with stathmin mAb (3352, Cell Signaling Technology, UK) or control IgG. After washing with 1X PBS, slides were reacted with the biotin-labeled second antibody and then visualized using an ultrasensitive streptavidin-peroxidases system (Maxim Biotech, Fuzhou, China). Semi-quantitative criteria of the stathmin immunoreaction were modified according to previous publications $(17,18)$. Immunostaining was scored as follows: 0 , negative; 1 , weak; 2 , moderate; and 3, strong staining. The percentage of stathmin staining area was graded as 0 , no positive staining; $1,<10 \% ; 2,10-50 \%$; or $3,50-100 \%$. The staining index was calculated as the multiples of staining intensity and staining area, as described $(18,19)$ The staining index $<1$ was considered as negative, while 1-4 as weak and $>4$ as strong.

Western blot analysis. Western blotting was performed as previously described (20). In briefly, protein extracted from cell lines and tissues specimens were separated using $12 \%$ SDS-PAGE, then transferred to polyvinylidene fluoride (PVDF) membranes (Millipore, Bedford, MA). Membranes were blocked by $10 \%$ skim milk in $1 \mathrm{X}$ PBS. The membranes were incubated with the primary antibodies against stathmin (ab52630, Abcam, UK) or $\beta$-actin (Cat. No. A-5316, Sigma, MO) in suitable dilutions. Secondary antibodies were antirabbit $\operatorname{IgG}$ and anti-mouse IgG, respectively. Signals were detected by chemiluminescence using the ECL kit.

siRNA transfection. The double-strand small interfering RNAs (siRNAs) were synthesized in duplex and purified forms using Genechem Co. (Shanghai, China). siRNAs targeting stathmin [5'-GAAACGAGAGCACGAGAAAtt-3' (forward) and 5'-UUUCUCGUGCUCUCGUUUCtt-3' (reverse)] and nonspecific scramble siRNA oligonucletide sequences [5'-UUC UCCGAACGUGUCACGUtt-3' (forward) and 5'-ACGUGAC ACGUUCGGAGAAtt-3' (reverse)] were transfected separately into KYSE30 and KYSE410 cells using Lipofectamine 2000 (Invitrogen, CA, USA) according to manufacturer's protocol. After $72 \mathrm{~h}$, proteins were extracted.

In vitro wound-healing assay. KYSE30, KYSE410 or EC0156 cells were incubated overnight yielding confluent monolayer for wounding. Wounds were scratched using a pipette tip, photographs were taken immediately (time $0 \mathrm{~h}$ ) and $24 \mathrm{~h}$ after wounding. The distance migrated by the cell monolayer to close the wounded area during this time period was measured. Photos were taken by Leica DMCI microscope (Leica, German) at x100 magnification.

Statistical analysis. Data were analyzed by SPSS 16.0 (SPSS Inc., Chicago, IL, USA). $\chi^{2}$ test was used to analyze the relationship between stathmin expression with clinicopathologic characteristics. P-values $<0.05$ were considered as statistically significant.

\section{Results}

The identification of stathmin in ESCC tissues. Firstly, we analyzed differientially expressed proteins using proteomic method between tumor and corresponding normal tissues in ESCC. Results showed stathmin was detected in all 2-DE gels 


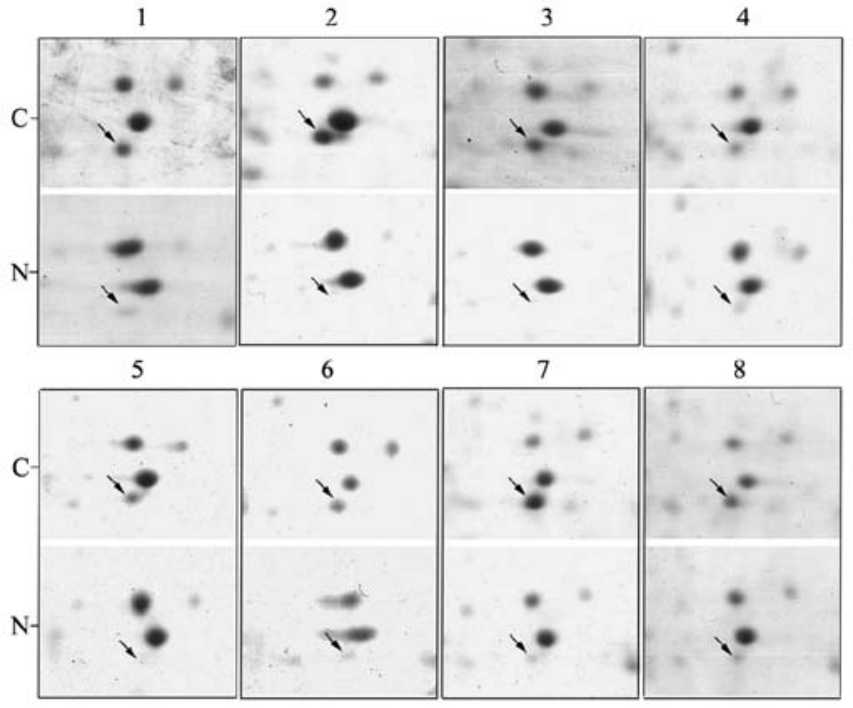

Figure 1. 2-DE images of spots identified as stathmin in 8 matched ESCC samples. N, the adjacent normal esophageal tissue; C, ESCC samples. The numbers represent the cases studied.

of the 8 pairs of ESCC tissues (Fig. 1), as an obvious differentially expressed spot.

Stathmin is overexpressed in ESCC tissues. Subsequently, we employed immunohistochemistry to analyze the expression of stathmin in 143 ESCC tissue microarray using the antistathmin antibody. Strong staining was seen in ESCC tissues, however, the normal tissues were weaker or negatively stained (Fig. 2). The strong staining of stathmin in cancer was $70.63 \%$ (101/143), while normal in $27.97 \%$ (40/143). In addition, the weak staining of stathmin in cancer was $20.28 \%(29 / 143)$, while normal in $15.38 \%(22 / 143)$. There was a significantly different staining mode between cancer and normal $(\mathrm{P}<0.05)$. Statistical analysis showed that overexpression of stathmin was significantly correlated with histological grade $(\mathrm{P}<0.05)$ (Table I). However, no correlation was found between stathmin expression and age, gender and lymph node metastasis $(\mathrm{P}>0.05)$ (Table I).

SiRNA-mediated reduction in stathmin expression resulted in impaired cell migration. We chose two ESCC cell lines (KYSE30 and KYSE410) as a model. SiRNA was employed to knockdown stathmin, and western blotting to detect the effect of siRNA. The results showed that the expression of stathmin was reduced after transfection of siRNA oligonucleotide for $72 \mathrm{~h}$ in KYSE30 and KYSE410 (Fig. 3A). Subsequently, wound-healing assay showed that the speed of wound recovery of siRNA stathmin was much slower than the scramble in both KYSE30 and KYSE410 (Fig. 3B). The results revealed that cell migration was impaired when deficient of stathmin.

The phosphorylation of stathmin reduced the motility of EC0156. Considering phosphorylation had been closely associtated with the function of stathmin, we wondered whether stathmin phosphorylation has influence on ESCC cell lines. We selected EC0156 for the next study.

EC0156 was treated with paclitaxel, a compound extracted from taxus plants, at gradient dosage from 0 to $1.6 \mu \mathrm{g} / \mathrm{ml}$. The results revealed that the $19 \mathrm{kDa}$ band of stathmin was decreased after paclitaxel treatment, whereas a new band at about $21 \mathrm{kDa}$ gradiently increased in a dose-dependent manner. We confirmed the new band was phosphorylated stathmin at Ser-16. The tendency increased dramatically between 0.1 and $1.6 \mu \mathrm{g} / \mathrm{ml}$, reaching a peak at a dosage of $0.8 \mu \mathrm{g} / \mathrm{ml}$. Conversely, KYSE30 and EC0156 without treatment had no

Table I. The correlation between stathmin expression and clinicopathological characteristics in ESCC specimens.

\begin{tabular}{|c|c|c|c|c|c|}
\hline \multirow[b]{2}{*}{ Characteristics } & \multirow[b]{2}{*}{ All cases $(n)(\%)$} & \multicolumn{3}{|c|}{ Stathmin } & \multirow[b]{2}{*}{$\mathrm{P}$-value } \\
\hline & & Negative $(\%)$ & Weak $(\%)$ & Strong (\%) & \\
\hline Age & & & & & $>0.05$ \\
\hline$\geq 60$ & $80(55.94)$ & $7(8.75)$ & $14(17.50)$ & $59(73.75)$ & \\
\hline$<60$ & $63(44.06)$ & $6(9.52)$ & $15(23.81)$ & $42(66.67)$ & \\
\hline Gender & & & & & $>0.05$ \\
\hline Male & $108(75.52)$ & $11(10.19)$ & $24(22.22)$ & $73(67.59)$ & \\
\hline Female & $35(24.48)$ & $2(5.71)$ & $5(14.29)$ & $28(80.00)$ & \\
\hline Tumor grade & & & & & 0.019 \\
\hline Well differentiated & $36(25.17)$ & $8(22.22)$ & $8(22.22)$ & $20(55.56)$ & \\
\hline Moderately differentiated & $80(55.94)$ & $5(6.25)$ & $16(20.00)$ & $59(73.75)$ & \\
\hline Poor differentiated & $27(18.89)$ & $0 \quad(0.00)$ & $5(18.52)$ & $22(81.48)$ & \\
\hline Lymph node metastasis ${ }^{\mathrm{a}}$ & & & & & $>0.05$ \\
\hline Present & $38(40.43)$ & $3(7.90)$ & $3(7.90)$ & $32(84.20)$ & \\
\hline Not present & $56(59.57)$ & $2(3.57)$ & $7(12.50)$ & $47(83.93)$ & \\
\hline
\end{tabular}

aOnly 94 cases have lymph node information. 

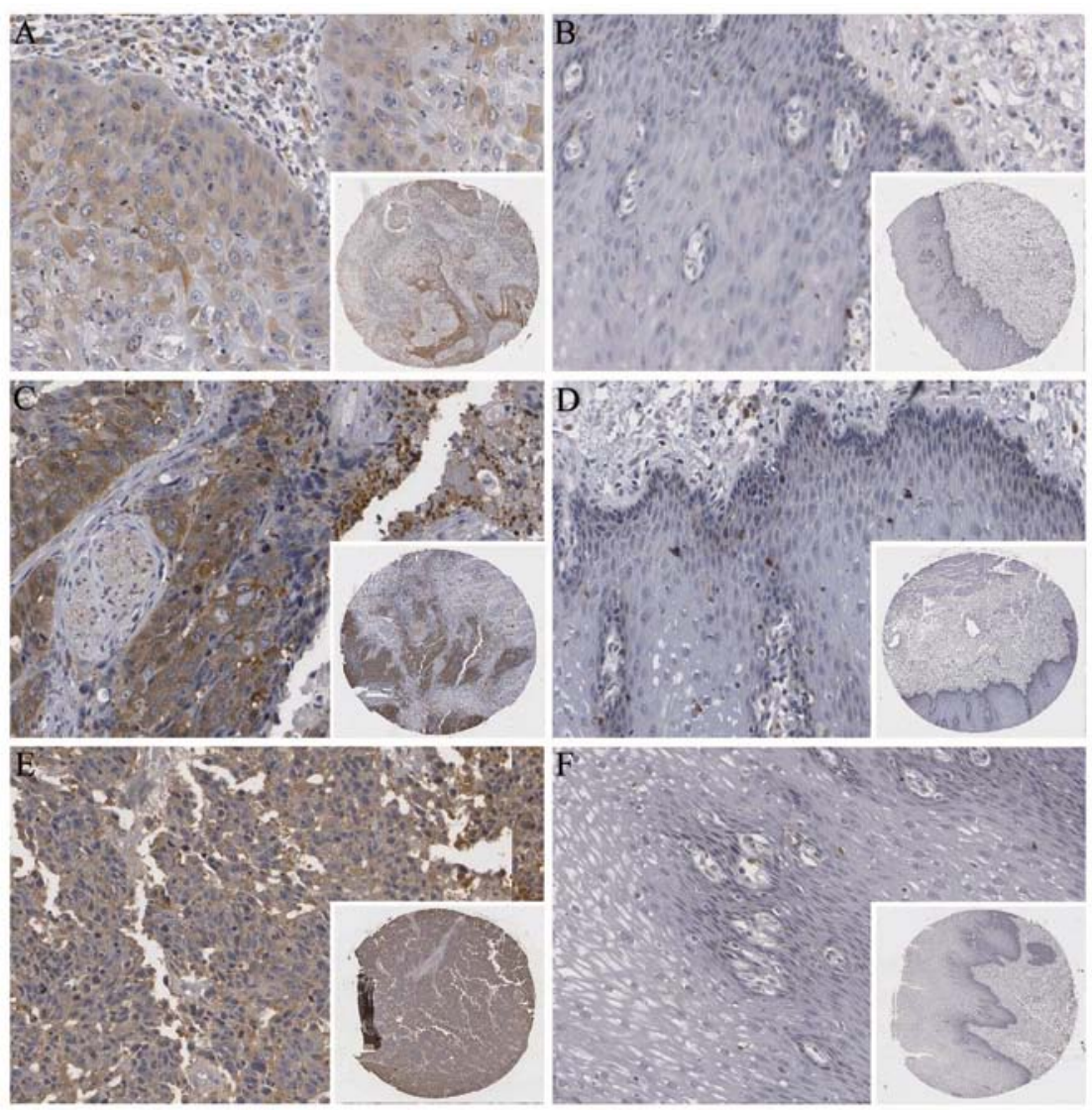

Figure 2. Representative immunohistochemistry staining of stathmin in ESCC specimens. (A, C and E) representative immunohistochemistry staining of cancer (x40), the lower right panels are shown at high magnification (x400), (A) well differentiated, (C) moderately differentiated, (E) poorly differentiated $(\mathrm{B}, \mathrm{D}$ and $\mathrm{F})$ representative immnohistological staining of normal $(\mathrm{x} 40)$. The lower right panels are shown high magnification $(\mathrm{x} 400)$.
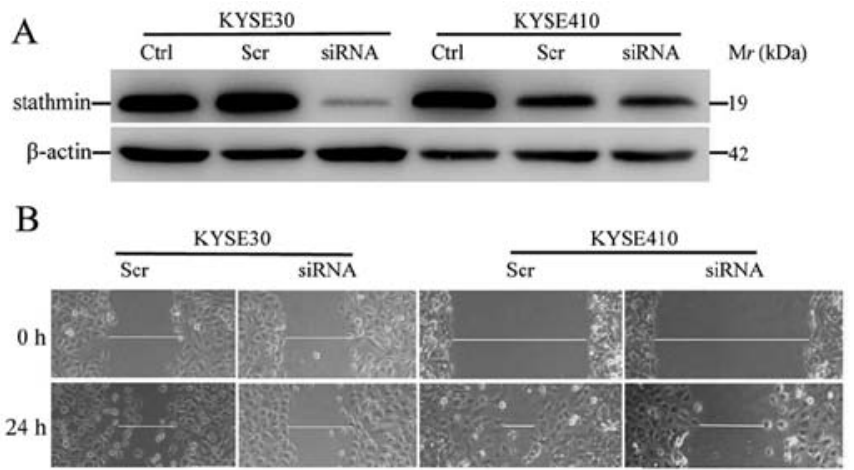

Figure 3. siRNA of stathmin in KYSE30 and KYSE 410 impair cell migration. (A) Analysis the effect of stathmin knockdown in KYSE30 and KYSE 410 using western blot analysis. (B) The wound-recovery result after knockdown in KYSE30 and KYSE410. Microtip scrape of the cultured cells, was then visualized under a microscope at x100 magnifcation. Ctrl, control; Scr, scramble.

changes (Fig. 4A). Besides, rounded cells were observed in most of the EC0156 through microscopic analysis (Fig. 4B). The number of cells appered slightly reduced. Wound-healing assay showed that the speed of wound recovery of EC0156 cells treated with pacitaxel was much slower than the control, suggesting that cell migration was impaired after stabilized phophorylation of stathmin (Fig. 4C).

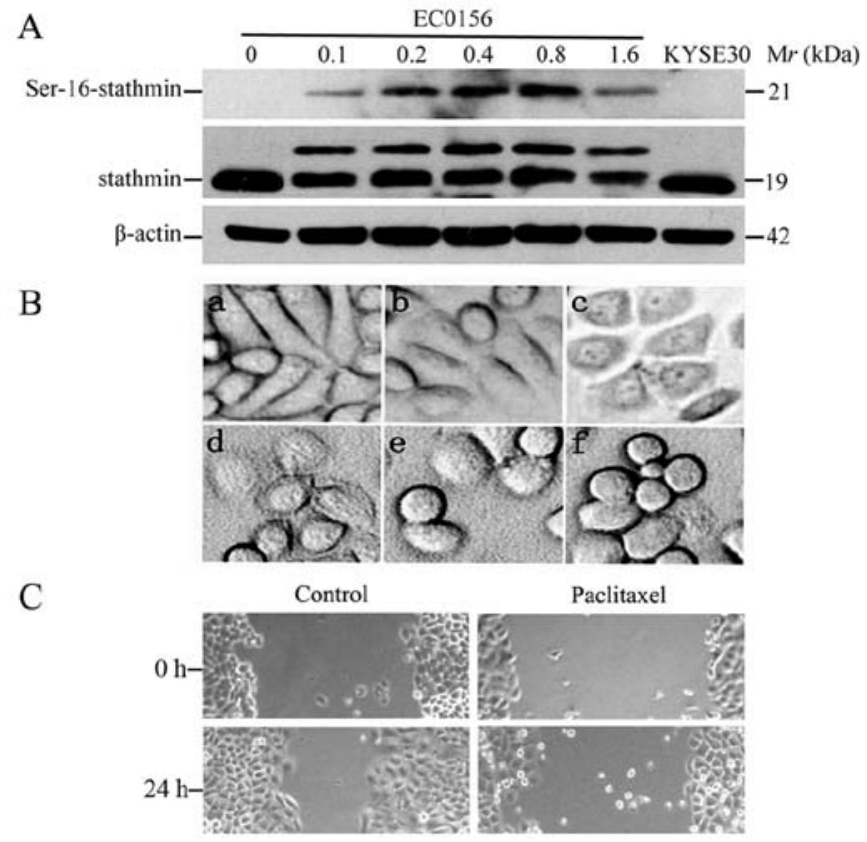

Figure 4. The phosphorylation of stathmin induced by paclitaxel impaired migration in EC0156. (A) Analysis the expression of stathmin in KYSE30 and KYSE 410 using western blot analysis after paclitaxel treatment. The treated paclitaxel dosage in EC0156 was $0.1,0.2,0.4,0.8$ and $1.6 \mu \mathrm{g} / \mathrm{ml}$ independently. (B) Cytological morphology variation after paclitaxel treatment. The paclitaxel dosage of treating EC0156 was $0.1,0.2,0.4,0.8$ and $1.6 \mu \mathrm{g} / \mathrm{ml}$, independently. (C) The wound-recovery results after paclitaxel treatment in EC0156. The paclitaxel dosage was $0.8 \mu \mathrm{g} / \mathrm{ml}$. 


\section{Discussion}

Previously, we analyzed differientially expressed proteins using proteomic methods between tumor and nomal tissues in ESCC. We found an obviously overexpressed spot in all tumor 2-DE gels, which was identified as stathmin. Stathmin is ubiquitous, highly conserved $19 \mathrm{kDa}$ cytosolic phosphoprotein that regulates microtubule dynamics (21).

We employed immunohistochemistry to detect the expression of stathmin in ESCC tissue microarray. Here we demonstrated strong expression in 77.14\% (108/140) of ESCC tissues. In addition, overexpression of stathmin was significantly correlated with histological grade $(\mathrm{P}<0.05)$. However, no correlation was found with age, gender and lymph node metastasis ( $\mathrm{P}>0.05)$. Wang et al revealed that the positive rate of stathmin in 75 ESCC samples was $81.3 \%$ and the relative contents of stathmin were significantly correlated with the differentiation degree, lymph node metastasis, invasive depth and TNM stage of ESCC (13). In addition, the basic tendency of stronger stathmin staining in ESCC was consistent with our previous study, which only used 13 ESCC samples (22). So far, many studies have demonstrated stathmin to be overexpressed in many human cancers, including mesothelioma tumor (11), malignant pheochromocytomas $(23,24)$, cervical carcinoma (25), primary nasopharyngeal carcinoma (26), gastric cancer (27), hepatocellular carcinoma $(28,29)$, medulloblastoma (30), endometrial cancer (18) and urothelial carcinoma (31). Above all, overexpression of stathmin was significantly correlated with clinical stage, tumor grade and lymph node metastasis in cancers, including cervical carcinoma, nasopharyngeal carcinoma, gastric cancer, hepatocellular carcinoma and endometrial cancer $(18,25-30)$. Moreover, stathmin may be regarded as a survival prognosis factor in ovarian cancer (32), endometrial cancer (18) and urothelial carcinoma (31). Our previous work, although the ESCC cases were only 13 for immunohistochemistry to analyze the expression of stathmin, we still found the expression of stathmin was overexpressed in ESCC tissue, but there was no correlation with tumor grades (22). In the present study, we expanded the ESCC case numbers for immunohistochemitry. Finally, we found overexpression of stathmin was significantly correlated with histological grade. However, the relationship between stathmin expression and lymph node metastasis did not replicate. There may be two reasons. The limited cases with lymph node metastasis in tissue microarray may lead to information being lost in our study. Another factor worth considered is the tiny difference of the pathologic criteria. In addition, the case number of the present study was much greater than the previous reports in ESCC.

We demonstrated that the speed of wound recovery in stathmin knockdown cells was much slower than the scramble in both KYSE30 and KYSE410 cells, suggesting cell migration was impaired when deficient of stathmin. Similar report exists in gastic cancer, in which cell invasion was significantly reduced by stathmin siRNA in the Matrigel invasion assay (27). Stathmin depletion with siRNA caused significant inhibition of lamellipodia formation, which directed by Pak1-WAVE2-kinesin complex (33). The lamellipodia formation may promote cell migration and invasion. Thus, stathmin depletion might inactivate lamellipodia formation leading to reduction of cell migration.
We demonstrated that the band at $19 \mathrm{kDa}$ of stathmin was decreased when EC0156 was treated by paclitaxel. Meantime, a new band at about $21 \mathrm{kDa}$ appeared in a dose-dependent manner. Subsequently, the new band was confirmed as phosphorylated stathmin at Ser-16. The basic function of stathmin was closely associated with its phosphorylated state. The stathmin-tubulin interaction was dependent on phosphorylation of stathmin (34). Rapidly switching phosphorylation of stathmin regulated microtubule assembly (21). Phosphorylation at Ser-16 and Ser-63 strongly reduced stathmin-tubulin complex formation (35). The effects of stathmin on dynamic instability were strongly attenuated by phosphorylation at Ser-16-and Ser-63 (6). Many protein kinases, including CDC2 (36), MAP (37), Auro B (38) and BGLF4 (39), can phosphorylate stathmin. Stathmin is known to undergo phosphorylation at Ser16 upon cell stimulation, such as paclitaxel at low concentration (40). Paclitaxel is an anticancer drug which interferes with microtubules (41). In the present study, we found paclitaxel may induce stathmin phosphorylation at Ser-16 and influenced the function of stathmin.

Through microscopic analysis, rounded cells were observed in most of EC0156 after the treatment with paclitaxel. The total number EC0156 appeared slightly reduced. Besides, the wound recovery speed of EC0156 treated with paclitaxel was diminished compared to the control, suggesting that cell migration may be impaired after stathmin phophorylation. It is possibly that stabilized phosphorylation of stathmin may disrupt microtubule dynamics and finally impaired the motility of EC0156, which were supported by other reports. Migration can be viewed as a repeated sequences of events that include formation of pseudopodia protrusions, attachment and translocation of the cell body in the direction of the new adhesion sites. The microtubule dynamics instability is important to generate an asymmetrical microtubules array (42). Stronger or long time stabilized phosphorylation of stathmin may impair the microtuble dynamics. Cernuda-Morollon et al found that T-cell receptor (TCR)-induced stathmin phosphorylation prevented T cell polarization on ICAM-1 by increasing Rac1 activity and reduced the migration of $\mathrm{T}$ cell and changed microtubule dynamics leading to loss of migratory polarity (43). Di Paolo et al revealed that phosphorylation at three sites (Ser-16, Ser-25, Ser-63) completely inhibited the tubulin binding capacity of stathmin (35). This phenomenon was also observated in cancer cells. Belletti et al revealed that Ser-16 phosphorylation of stathmin enhanced sarcoma cell adhesion and inhibited sarcoma cell motility by mutant methods (44). The present study revealed that paclitaxel may act as a stimulus factor to induce the phosphorylaiton of stathmin leading to impairment of microtubule dynamics. Our observations provide new evidence for understanding the interaction between stathmin phosphorylation and microtubules.

Our observation at cell level was not in accordance with immunohistochemistry analysis. Except for information lost or cancer diversity, different microenvironment between cultured cells and tissues may also contribute. In summary, stathmin was overexpressed in ESCC tissues and overexpression of stathmin was significantly correlated with histological grade. In addition, deficiency or stabilized phosphorylation of stathmin both impaired the migration of ESCC cells. The 
present study might highlight the potential of stathmin in the therapy and diagnosis of ESCC.

\section{Acknowledgements}

We thank Dr Rong Wang in Mount Sinai School of Medicine and Dr Si-qi Liu in Beijing Genomics Institute for critical suggestion. This study was supported by National High-Tech R\&D Program (No. 2012AA020206, 2012AA02A503), SKPBR (No. 2011CB910703) and NSFC (No. 91029725, 81071789, 81071811) of China.

\section{References}

1. Jemal A, Siegel R, Ward E, Hao Y, Xu J and Thun MJ: Cancer statistics, 2009. CA Cancer J Clin 59: 225-249, 2009.

2. Lin DC, Du XL and Wang MR: Protein alterations in ESCC and clinical implications: a review. Dis Esophagus 22: 9-20, 2009.

3. Steinmetz MO: Structure and thermodynamics of the tubulinstathmin interaction. J Struct Biol 158: 137-147, 2007.

4. Belmont LD and Mitchison TJ: Identification of a protein that interacts with tubulin dimers and increases the catastrophe rate of microtubules. Cell 84: 623-631, 1996.

5. Cassimeris L: The oncoprotein 18/stathmin family of microtubule destabilizers. Curr Opin Cell Biol 14: 18-24, 2002.

6. Manna T, Thrower DA, Honnappa S, Steinmetz MO and Wilson L: Regulation of microtubule dynamic instability in vitro by differentially phosphorylated stathmin. J Biol Chem 284 15640-15649, 2009.

7. Marklund U, Larsson N, Gradin HM,Brattsand G and Gullberg M: Oncoprotein 18 is a phosphorylation-responsive regulator of microtubule dynamics. EMBO J 15: 5290-5298, 1996.

8. Amayed P, Pantaloni D and Carlier MF: The effect of stathmin phosphorylation on microtubule assembly depends on tubulin critical concentration. J Biol Chem 277: 22718-22724, 2002.

9. Niethammer P, Bastiaens P and Karsenti E: Stathmin-tubulin interaction gradients in motile and mitotic cells. Science 303: 1862-1866, 2004.

10. Liu Z, Lu H, Shi H, et al: PUMA overexpression induces reactive oxygen species generation and proteasome-mediated stathmin degradation in colorectal cancer cells. Cancer Res 65: 1647-1654, 2005.

11. Kim JY, Harvard C, You L, et al: Stathmin is overexpressed in malignant mesothelioma. Anticancer Res 27: 39-44, 2007.

12. Nakashima D, Uzawa K, Kasamatsu A, et al: Protein expression profiling identifies maspin and stathmin as potential biomarkers of adenoid cystic carcinoma of the salivary glands. Int J Cancer 118: 704-713, 2006.

13. Wang F, Wang LX, He W, Zhu LN, Zhao PR and Fan QX: Expression of stathmin in esophageal squamous cell carcinoma and its biological significance. Nan Fang Yi Ke Da Xue Xue Bao 30: 1552-1557, 2010 (In Chinese).

14. Wang X, Fan QX, Zhao PR and Wang F: Expression and significance of stathmin in esophageal squamous cell carcinoma. J Basic Clin Oncol 120: 372-374, 2007.

15. Wang F, Wang LX, Fan QX and Zhao PR: Inhibitory effects of ASODN of stathmin gene on cultured Eca109 cell lines. J Zhengzhou University (Medical Sciences) 43: 414-418, 2008.

16. Wang Q, Xu Y, Zhao X, et al: A facile one-step in situ functionalization of quantum dots with preserved photoluminescence for bioconjugation. J Am Chem Soc 129: 6380-6381, 2007.

17. Kouzu Y, Uzawa K, Koike H, et al: Overexpression of stathmin in oral squamous-cell carcinoma: correlation with tumour progression and poor prognosis. Br J Cancer 94: 717-723, 2006.

18. Trovik J, Wik E, Stefansson IM, et al: Stathmin overexpression identifies high risk patients and lymph node metastasis in endometrial cancer. Clin Cancer Res 17: 3368-3377, 2011.

19. Salvesen HB, Das S and Akslen LA: Loss of nuclear p16 protein expression is not associated with promoter methylation but defines a subgroup of aggressive endometrial carcinomas with poor prognosis. Clin Cancer Res 6: 153-159, 2000.

20. Sun Y, Mi W, Cai J, et al: Quantitative proteomic signature of liver cancer cells: tissue transglutaminase 2 could be a novel protein candidate of human hepatocellular carcinoma. J Proteome Res 7: 3847-3859, 2008.
21. Rana S, Maples PB, Senzer N and Nemunaitis J: Stathmin 1: a novel therapeutic target for anticancer activity. Expert Rev Anticancer Ther 8: 1461-1470, 2008

22. Liu F, Liu F, Sun YL and Zhao XH: Significance of STMN1 expression in esophageal squamous cell carcinoma. World Chin J Digestol 18: 1306-1312, 2010.

23. Sadow PM, Rumilla KM, Erickson LA and Lloyd RV: Stathmin expression in pheochromocytomas, paragangliomas, and in other endocrine tumors. Endocr Pathol 19: 97-103, 2008.

24. Bjorklund P, Cupisti K, Fryknas M, et al: Stathmin as a marker for malignancy in pheochromocytomas. Exp Clin Endocrinol Diabetes 118: 27-30, 2010.

25. Xi W, Rui W, Fang L, Ke D, Ping G and Hui-Zhong Z: Expression of stathmin/op18 as a significant prognostic factor for cervical carcinoma patients. J Cancer Res Clin Oncol 135: 837-846, 2009.

26. Cheng AL, Huang WG, Chen ZC, et al: Identification of novel nasopharyngeal carcinoma biomarkers by laser capture microdissection and proteomic analysis. Clin Cancer Res 14: 435-445, 2008.

27. Jeon TY, Han ME, Lee YW, et al: Overexpression of stathmin1 in the diffuse type of gastric cancer and its roles in proliferation and migration of gastric cancer cells. Br J Cancer 102: 710-718, 2010.

28. Yuan RH, Jeng YM, Chen HL, et al: Stathmin overexpression cooperates with p53 mutation and osteopontin overexpression, and is associated with tumour progression, early recurrence, and poor prognosis in hepatocellular carcinoma. J Pathol 209: $549-558,2006$.

29. Gan L, Guo K, Li Y, et al: Up-regulated expression of stathmin may be associated with hepatocarcinogenesis. Oncol Rep 23: 1037-1043, 2010.

30. Kuo MF, Wang HS, Kuo QT, et al: High expression of stathmin protein predicts a fulminant course in medulloblastoma. J Neurosurg Pediatr 4: 74-80, 2009.

31. Lin WC, Chen SC, Hu FC, et al: Expression of stathmin in localized upper urinary tract urothelial carcinoma: correlations with prognosis. Urology 74: 1264-1269, 2009.

32. Su D, Smith SM, Preti M, et al: Stathmin and tubulin expression and survival of ovarian cancer patients receiving platinum treatment with and without paclitaxel. Cancer 115: 2453-2463, 2009.

33. Takahashi K and Suzuki K: Membrane transport of WAVE2 and lamellipodia formation require Pak1 that mediates phosphorylation and recruitment of stathmin/Op18 to Pak1-WAVE2-kinesin complex. Cell Signal 21: 695-703, 2009.

34. Ravelli RB, Gigant B, Curmi PA, et al: Insight into tubulin regulation from a complex with colchicine and a stathmin-like domain. Nature 428: 198-202, 2004.

35. Di Paolo G, Antonsson B, Kassel D, Riederer BM and Grenningloh G: Phosphorylation regulates the microtubuledestabilizing activity of stathmin and its interaction with tubulin. FEBS Lett 416: 149-152, 1997.

36. Moreno FJ and Avila J: Phosphorylation of stathmin modulates its function as a microtubule depolymerizing factor. Mol Cell Biochem 183: 201-209, 1998

37. Antonsson B, Kassel DB, Ruchti E and Grenningloh G: Differences in phosphorylation of human and chicken stathmin by MAP kinase. J Cell Biochem 80: 346-352, 2001.

38. Gadea BB and Ruderman JV: Aurora B is required for mitotic chromatin-induced phosphorylation of Op18/Stathmin. Proc Natl Acad Sci USA 103: 4493-4498, 2006.

39. Chen PW, Lin SJ, Tsai SC, et al: Regulation of microtubule dynamics through phosphorylation on stathmin by Epstein-Barr virus kinase BGLF4. J Biol Chem 285: 10053-10063, 2010.

40. Andersen SS: Spindle assembly and the art of regulating microtubule dynamics by MAPs and Stathmin/Op18. Trends Cell Biol 10: 261-267, 2000.

41. Mistry SJ, Bank A and Atweh GF: Synergistic antiangiogenic effects of stathmin inhibition and taxol exposure. Mol Cancer Res 5: 773-782, 2007.

42. Honore S, Pasquier E and Braguer D: Understanding microtubule dynamics for improved cancer therapy. Cell Mol Life Sci 62: 3039-3056, 2005.

43. Cernuda-Morollon E, Millan J, Shipman M, Marelli-Berg FM and Ridley AJ: Rac activation by the T-cell receptor inhibits T cell migration. PLoS One 5: e12393, 2010.

44. Belletti B, Nicoloso MS, Schiappacassi M, et al: Stathmin activity influences sarcoma cell shape, motility, and metastatic potential. Mol Biol Cell 19: 2003-2013, 2008. 